\title{
DNA Polymerase-Catalyzed DNA Network Growth
}

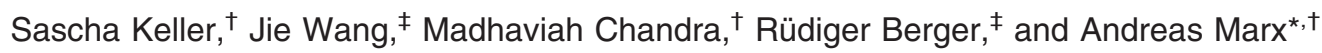 \\ Department of Chemistry and Konstanz, Research School Chemical Biology, Universität Konstanz, 78457 Konstanz, \\ Germany, and Max Planck Institute for Polymer Research, 55128 Mainz, Germany
}

\section{E-mail: andreas.marx@uni-konstanz.de}

DNA is the fundamental genetic material for the storage and transfer of information from one generation to another. The Watson-Crick base pairing properties of DNA is an advantageous phenomenon that has been exploited in the usage of DNA as scaffold for directed self-organization to form nanometer-sized objects in a desirable fashion. ${ }^{1}$ In the first report, Seeman proposed ordered arrays of DNA with branched DNA (bDNA) building blocks that naturally exist in cellular DNA metabolism. ${ }^{2}$ Since then, several reports have appeared in literature that describe the generation of bDNAs with a variable number of arms to selfassemble with predesigned architectures. ${ }^{1}$ One prospective advantage of DNA for nanoconstruction is the inherent potential for further manipulations by DNA modifying enzymes. This issue is only beginning to be fully explored. DNA ligases have been employed to covalently link DNA strands to form nanometer-sized objects $^{3}$ or DNA-based networks ${ }^{4}$ that form hydrogels or can be exploited in diagnostics. DNA polymerase-catalyzed nanoconstruction was reported for rolling circle amplification that holds the potential for enzymatic amplification of DNA structures with high fidelity. ${ }^{5}$ Additionally, the DNA polymerase-synthesized singlestranded DNA that folds into an octahedron with the assistance of scaffolding DNA oligomers was described. ${ }^{6}$ Besides these biologically inspired bDNA constructs several covalently and noncovalently linked branching points were reported, and their employment in nanoconstruction was demonstrated. ${ }^{7}$ However, these strategies are only scarcely exploited for enzymatic synthesis and manipulations despite the potential for the introduction of additional functionalization through chemical modifications within the scaffolds.

Herein we report for the first time the construction of threedimensional DNA-based networks that can be generated and amplified by the DNA polymerase chain reaction (PCR). The approach is based on the combination of covalently connected bDNA Y-motifs that act as primer strands in PCR. The distance between the branched points can be tuned by use of templates with different length ranging from oligonucleotides to entire genes. Our approach to develop PCR-amplifiable three-dimensional DNA networks is based on branched sense (I) and reversed primer (II) strands and suitable templates that are designed to be used by DNA polymerases as primers for enzymatic amplification (Figure 1). The branch primer strands were synthesized as previously described ${ }^{7 f}$ using the branch point 1 and phosphoramidites for standard $3^{\prime} \rightarrow 5^{\prime}$ solid phase synthesis as well as those for $5^{\prime} \rightarrow 3^{\prime}$ synthesis allowing all branches to have the same sequence and to terminate with a free 3 '-OH required for processing by DNA polymerases. In the first step of our approach the branched primer $\mathbf{I}$ is expected to anneal with the template III and be extended by a DNA polymerase in the presence of all four dNTPs to an enlarged Y-motif IV (Figure 1.). IV harbors binding sites for the second branched primer II required for amplifications by PCR. At the beginning of the next

\footnotetext{
Universität Konstanz.

* Max Planck Institute.
}

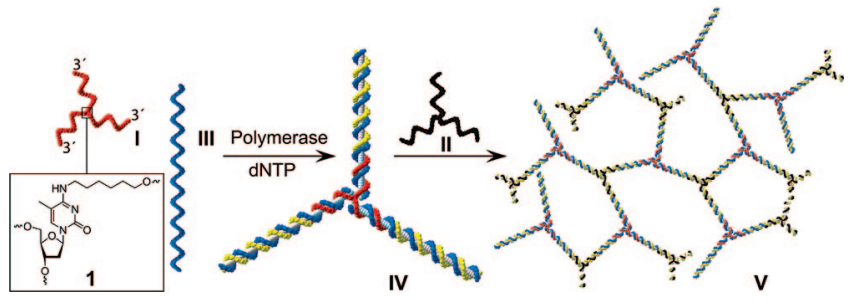

Figure 1. Schematic depiction of the generation of DNA networks by PCR amplification using branched primer strands.

PCR cycle, the denaturing step separates IV and opens a location for I and II to anneal. At this step, all ends bear branched primers that allow the formation and growth of DNA networks $\mathbf{V}$ by the PCR.

To investigate whether our approach is indeed practicable we first applied branched primer Ia and IIa that bear 20nt long branches and used single stranded DNA templates of 90, 80, 70nt lengths, respectively. All PCR experiments were conducted in parallel using nonbranched primer strands with the same sequence under identical conditions. The later PCR reactions yielded products that migrated with expected mobility by agarose gel electrophoresis analysis (Figure 2A, lanes 1, 3, 5). However, when the branched primer strands Ia and IIa were employed using the same conditions the formation of reaction products of higher complexity and lower mobility in gel electrophoresis were observed (Figure 2A, lanes 2, $4,6)$. Interestingly, the size of the generated constructs varied with the length of the used template. To gain evidence that indeed amplification resulted from template usage and not artifacts or false amplification we conducted a series of experiments. First, no amplification products were formed in the absence of template under the identical conditions (data not shown). Additionally, by correct template usage, DNA networks are generated that contain a single PstI restriction site in one extended DNA branch. Indeed, treatment with PstI endonuclease resulted in two discrete DNA constructs that migrated with the expected mobility (Figure 2B). These findings indicate that indeed DNA networks were formed by amplification.

Our approach holds the potential for further functionalizations through incorporation of modified building blocks into the DNA network. Since DNA polymerases are known to tolerate several modifications of the dNTP substrates, ${ }^{8}$ we next investigated whether dCTP can be partially replaced by a Cy3-labeled analogue to generate DNA networks that contain covalent DNA-bound dyes. Indeed we found that increasing the amounts of the dye-labeled nucleotide in the above depicted PCR reactions using Ia and IIa led to the formation of DNA networks that fluoresce upon excitation showing the incorporation of the fluorescent dye (Figure 2C). The finding that additional complexity can be introduced in the DNA networks by the enzymatic reaction opens the possibility for further construction and conjugation based on the depicted networks. 

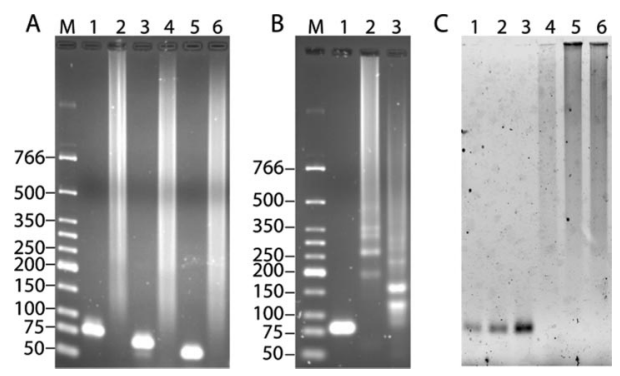

Figure 2. Generation and agarose gel electrophoresis analysis of DNA networks by PCR. (A) Generation of DNA networks by PCR with varied DNA templates and Ia and IIa in comparison to nonbranched primers: (lane M) DNA ladder; (lanes 1, 3, 5) PCR reaction products from nonbranched primers and 90nt, 80nt, and 70nt long templates, respectively; (lanes 2, 4, 6) PCR reaction products from Ia and IIa and 90nt, 80nt, and 70nt templates, respectively. (B) Restriction digest: (lane M) DNA ladder; (lane 1) PCR reaction products from nonbranched primers and 90nt templates; (lane 2) same as lane 1 with Ia and IIa instead of nonbranched primers; (lane 3) reaction product from lane 2 after Pst I digest. (C) PCR reaction products from 90nt template and nonbranched primers (lanes 1-3) or branched primers (lanes 4-6), respectively, in the presence of increasing amounts $(10 \%, 20 \%, 30 \%)$ of Cy3-labeled dCTP. For experimental details see Supporting Information.

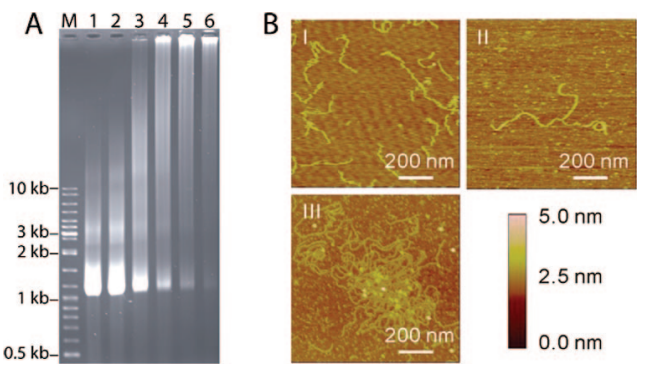

Figure 3. Generation and analysis of DNA networks from 1062nt template. (A) Generation of DNA networks by PCR with 1062 nt DNA template and branched primers Ib and IIb: (lane M) DNA ladder; (lanes 1-6) PCR reaction products formed at increasing annealing temperatures $\left(51.0^{\circ} \mathrm{C}\right.$, $52.7^{\circ} \mathrm{C}, 56.8^{\circ} \mathrm{C}, 61.1^{\circ} \mathrm{C}, 65.3^{\circ} \mathrm{C}, 68.6^{\circ} \mathrm{C}$, respectively). (B) AFM analysis of DNA structures: (I) structures occurring resulting from nonbranched primers; (II) first cycle-reaction product using Ib and IIb; (III) DNA networks generated after 40 PCR cycles. For comparison, the lateral scales of all images are $200 \mathrm{~nm}$ and the height scales are $5 \mathrm{~nm}$.

Next, we investigated whether the stretches between the branching units can be enlarged by usage of enlarged DNA and investigated the 1062nt open reading frame of human DNA polymerase $\beta$. When using the branched primer Ib and IIb (sequences see Supporting Information) the formation of DNA network was depending on the optimal annealing temperature during PCR cycling (Figure $3 \mathrm{~A}$ ). While at $51^{\circ} \mathrm{C}$ DNA network formation was hardly observed, at $69{ }^{\circ} \mathrm{C}$ DNA networks with low electrophoretic mobility were yielded. Again, no amplification products were formed in the absence of template under the identical conditions (data not shown). To gain more insight into the formed structures we imaged the primers and networks in liquid on a mica surface by atomic force microscopy (AFM) operated in soft tapping mode. ${ }^{9}$ As a control, standard linear primer strands used in PCR were investigated (Figure 3B, I). Linear primer strands are considerably flexible resulting in linear or coiled structures having a height ranging from 1.3 to $1.8 \mathrm{~nm}$ and a width of about $15 \mathrm{~nm}$. The deviation in height compared to the theoretic diameter of dsDNA $(2 \mathrm{~nm})$ is attributed to the force of the scanning tip and the surface resulting in flattened objects. ${ }^{10}$ The increased width is given by the broadening effect of the finite size of the AFM tip. ${ }^{11}$ For branched primer strands Ib and II $\mathbf{I b}$, we found extended branched primer strands that were formed after the first cycle of amplification, confirming the formation of the DNA structure IV during the formation of DNA networks (Figure 3B, II). The lengths of each of the three DNA branches are consistent with the expected value which is approximately $370 \mathrm{~nm}$. Finally, further AFM investigations show the formation of DNA networks in variable shapes (Figure $3 \mathrm{~B}, \mathrm{III})$. These irregular shapes may be caused by the collapse of the three-dimensional structure resulting from the interaction with the mica surface. Nevertheless, the AFM visualization of DNA networks on the mica surface verified the capability of the developed method which was further supported by overview AFM scans and a height analysis of DNA junctions (see Supporting Information).

In conclusion, we describe a new approach for the generation of DNA networks by PCR amplification using branched primer constructs. The approach is flexible, allowing tuning of the meshes of the network by variation of the template size. Additionally, further diversification can be introduced by the employment of chemically modified nucleotides in PCR allowing the introduction of functionalities and reporter moieties.

Acknowledgment. This work was partially supported by the DFG Sonderforschungsbereich 625 "From Single Molecules to Nanoscopically Structured Materials."

Supporting Information Available: Experimental details, DNA sequences, and further AFM pictures. This material is available free of charge via the Internet at http://pubs.acs.org.

\section{References}

(1) (a) Seeman, N. C. Nature 2003, 421, 427-431. (b) Feldkamp, U.; Niemeyer, C. M. Angew. Chem., Int. Ed. 2006, 45, 1856-1876. (c) Lin, C. X.; Liu, Y.; Rinker, S.; Yan, H. ChemPhysChem 2006, 7, 1641-1647. (d) Deng, Z. X.; Lee, S. H.; Mao, C. D. J. Nanosci. Nanotechnol. 2005, 5, 19541963.

(2) Seeman, N. C. J. Theor. Biol. 1982, 99, 237-247.

(3) (a) Chen, J. H.; Seeman, N. C. Nature 1991, 350, 631-633. (b) Zhang, Y. W.; Seeman, N. C. J. Am. Chem. Soc. 1994, 116, 1661-1669. (c) Goodman, R. P.; Schaap, I. A. T.; Tardin, C. F.; Erben, C. M.; Berry, R. M.; Schmidt, C. F.; Turberfield, A. J. Science 2005, 310, 1661-1665.

(4) (a) Um, S. H.; Lee, J. B.; Park, N.; Kwon, S. Y.; Umbach, C. C.; Luo, D. Nat. Mater. 2006, 5, 797-801. (b) Li, Y. G.; Cu, Y. T. H.; Luo, D. Nat. Biotechnol. 2005, 23, 885-889. (c) Li, Y. G.; Tseng, Y. D.; Kwon, S. Y.; d'Espaux, L.; Bunch, J. S.; McEuen, P. L.; Luo, D. Nat. Mater. 2004, 3, $38-42$.

(5) (a) Lin, C. X.; Wang, X.; Liu, Y.; Seeman, N. C.; Yan, H. J. Am. Chem. Soc. 2007, 129, 14475-14481. (b) Lin, C. X.; Xie, M. Y.; Chen, J. J. L.; Liu, Y: Yan, H. Angew. Chem., Int. Ed. 2006, 45, 7537-7539.

(6) Shih, W. M.; Quispe, J. D.; Joyce, G. F. Nature 2004, 427, 618-621.

(7) (a) Scheffler, M.; Dorenbeck, A.; Jordan, S.; Wustefeld, M.; von Kiedrowski, G. Angew. Chem., Int. Ed. 1999, 38, 3312-3315. (b) Eckardt, L. H.; Naumann, K.; Pankau, W. M.; Rein, M.; Schweitzer, M.; Windhab, N.; von Kiedrowski, G. Nature 2002, 420, 286. (c) Stewart, K. M.; McLaughlin, L. W. J. Am. Chem. Soc. 2004, 126, 2050-2057. (d) Gothelf, K. V.; Thomsen, A.; Nielsen, M.; Cló, E.; Brown, R. S. J. Am. Chem. Soc. 2004, 126, 1044-1046. (e) Aldaye, F. A.; Sleiman, H. F. Angew. Chem., Int. Ed. 2006, 45, 2204-2209. (f) Chandra, M.; Keller, S.; Gloeckner, C.; Bornemann, B.; Marx, A. Chem. Eur. J. 2007, 13, 3558-3564. (g) Zimmermann, J.; Cebulla, M. R. J.; Mönningshoff, S.; von Kiedrowski, G. Angew. Chem., Int. Ed. 2008, 47, 3626-3630.

(8) Beaucage, S. L. In Comprehensive Natural Products Chemistry; Kool, E. T., Ed.; Pergamon Press: 1999; Vol. 7, pp 153-249.

(9) (a) Hansma, H. G. Annu. Rev. Phys. Chem. 2001, 52, 71-92. (b) Ding, K.; Alemdaroglu, F. E.; Börsch, M.; Berger, R.; Herrmann, A. Angew. Chem., Int. Ed. 2007, 46, 1172-1175.

(10) Magonov, S. N.; Elingsa, V.; Whangbo, M.-H. Surf. Sci. 1997, 375, L385L391.

(11) Bustamante, C.; Keller, D.; Yang, G. L. Curr. Opin. Struct. Biol. 1993, 3, $363-372$. 